\title{
Toward Optimization of Imaging System and Lymphatic Tracer for Near-Infrared Fluorescent Sentinel Lymph Node Mapping in Breast Cancer
}

\author{
J. Sven D. Mieog, MD ${ }^{1}$, Susan L. Troyan, MD ${ }^{2}$, Merlijn Hutteman, MSc ${ }^{1,3}$, Kevin J. Donohoe, MD $^{4}$, Joost R. van der \\ Vorst, MD ${ }^{1}$, Alan Stockdale, MEd ${ }^{3}$, Gerrit-Jan Liefers, $\mathrm{MD}$, $\mathrm{PhD}^{1}$, Hak Soo Choi, $\mathrm{PhD}^{3}$, Summer L. Gibbs-Strauss, \\ $\mathrm{PhD}^{3}$, Hein Putter, $\mathrm{PhD}^{5}$, Sylvain Gioux, $\mathrm{PhD}^{3}$, Peter J. K. Kuppen, $\mathrm{PhD}^{1}$, Yoshitomo Ashitate, $\mathrm{MD}^{3}$, Clemens \\ W. G. M. Löwik, PhD $^{6}$, Vincent T. H. B. M. Smit, MD, PhD ${ }^{7}$, Rafiou Oketokoun, $\mathrm{MS}^{3}$, Long H. Ngo, PhD $^{8}$, \\ Cornelis J. H. van de Velde, MD, PhD ${ }^{1}$, John V. Frangioni, MD, PhD $^{3,4}$, and Alexander L. Vahrmeijer, MD, PhD $^{1}$ \\ ${ }^{1}$ Department of Surgery, Leiden University Medical Center, Leiden, The Netherlands; ${ }^{2}$ Breast Care Center, Department of \\ Surgery, Beth Israel Deaconess Medical Center, Boston, MA; ${ }^{3}$ Division of Hematology/Oncology, Department of \\ Medicine, Beth Israel Deaconess Medical Center, Boston, MA; ${ }^{4}$ Department of Radiology, Beth Israel Deaconess Medical \\ Center, Boston, MA; ${ }^{5}$ Department of Medical Statistics, Leiden University Medical Center, Leiden, The Netherlands; \\ ${ }^{6}$ Department of Endocrinology, Leiden University Medical Center, Leiden, The Netherlands; ${ }^{7}$ Department of Pathology, \\ Leiden University Medical Center, Leiden, The Netherlands; ${ }^{8}$ Division of General Medicine, Department of Medicine, \\ Beth Israel Deaconess Medical Center, Boston, MA
}

\begin{abstract}
Background. Near-infrared (NIR) fluorescent sentinel lymph node (SLN) mapping in breast cancer requires optimized imaging systems and lymphatic tracers.

Materials and Methods. A small, portable version of the FLARE imaging system, termed Mini-FLARE, was developed for capturing color video and two semi-independent channels of NIR fluorescence (700 and $800 \mathrm{~nm}$ ) in real time. Initial optimization of lymphatic tracer dose was
\end{abstract}

J. Sven D. Mieog, S. L. Troyan, and M. Hutteman contributed equally to the study and share first authorship.

Clinical Trial Registration: ClinicalTrial.gov NCT00721370 and The Netherlands Trial Register NTR2084.

Electronic supplementary material The online version of this article (doi:10.1245/s10434-011-1566-x) contains supplementary material, which is available to authorized users.

(C) The Author(s) 2011. This article is published with open access at Springerlink.com

First Received: 17 November 2010;

Published Online: 1 March 2011

J. V. Frangioni, MD, PhD

e-mail: jfrangio@bidmc.harvard.edu

A. L. Vahrmeijer, MD, PhD

e-mail: a.1.vahrmeijer@lumc.nl performed using 35-kg Yorkshire pigs and a 6-patient pilot clinical trial. More refined optimization was performed in 24 consecutive breast cancer patients. All patients received the standard of care using ${ }^{99 \mathrm{~m}}$ Technetium-nanocolloid and patent blue. In addition, $1.6 \mathrm{ml}$ of indocyanine green adsorbed to human serum albumin (ICG:HSA) was injected directly after patent blue at the same location. Patients were allocated to 1 of 8 escalating ICG:HSA concentration groups from 50 to $1000 \mu \mathrm{M}$.

Results. The Mini-FLARE system was positioned easily in the operating room and could be used up to $13 \mathrm{in}$. from the patient. Mini-FLARE enabled visualization of lymphatic channels and SLNs in all patients. A total of 35 SLNs (mean $=1.45$, range $1-3$ ) were detected: 35 radioactive (100\%), 30 blue (86\%), and 35 NIR fluorescent (100\%). Contrast agent quenching at the injection site and dilution within lymphatic channels were major contributors to signal strength of the SLN. Optimal injection dose of ICG:HSA ranged between 400 and $800 \mu \mathrm{M}$. No adverse reactions were observed.

Conclusions. We describe the clinical translation of a new NIR fluorescence imaging system and define the optimal ICG:HSA dose range for SLN mapping in breast cancer.

Sentinel lymph node (SLN) mapping, as introduced for the management of breast cancer by Giuliano et al., is 
currently regarded as the standard of care for staging of the axilla. $^{1,2}$ In general, a combination of radioactive colloid and blue dye is used. However, this exposes patients and caregivers to ionizing radiation, and blue dyes cannot be seen through skin and fatty tissue. Nonetheless, in recent trials, SLN identification rates of $95-97 \%$ are achieved when using this combination, and use of only 1 agent results in significantly lower identification rates. ${ }^{3-6}$

Recent preclinical and clinical data have demonstrated that near-infrared (NIR) fluorescence imaging using the NIR fluorescence agent indocyanine green (ICG) enables real-time transcutaneous and intraoperative visualization of lymphatic channels and detection of the SLN. ${ }^{7-14}$ Therefore, NIR fluorescence imaging could provide an alternative for, or an addition to, conventional techniques used for SLN mapping. However, to date, clinical data are lacking a direct comparison of NIR fluorescence to the combination of radioactive tracer and blue dye. Also, even though ICG is the only fluorescent agent currently available in the clinic, it is not an optimal lymphatic tracer. Previous preclinical work has demonstrated that adsorption of ICG to human serum albumin (HSA, complex is ICG:HSA) increases the fluorescence intensity and the hydrodynamic diameter, thereby providing improved detection and better retention in the SLN. ${ }^{15}$

Recently, first in-human clinical testing of ICG:HSA was performed. ${ }^{14}$ The fluorescence-assisted resection and exploration (FLARE) imaging system, developed by our group and used in the trial, is a general-purpose optical imaging platform that provides the surgeon with 2 independent NIR fluorescence channels (centered at 700 and $800 \mathrm{~nm}$ ) to see otherwise invisible structures within the surgical field. ${ }^{14,16}$ The first generation of FLARE was large, expensive to build, and had a heavy imaging head that required a specially designed articulated arm. ${ }^{14}$ This prevented shipment of FLARE to researchers around the world, and therefore impeded efficient scientific investigation. During the first in-human clinical testing of FLARE, it also became apparent that contrast agents for SLN mapping were not yet optimal. The goals of the present study were to develop a miniaturized version of FLARE, termed Mini-FLARE, and to determine optimal lymphatic tracer dosing for SLN mapping in breast cancer.

\section{METHODS}

\section{Mini-FLARE Imaging System}

A detailed description of the Mini-FLARE imaging system and in vitro characterization is provided in Supplementary Data. For sterile usage in the operating room, a 0.118 -in. thick acrylic splash shield having $95 \%$ optical transmission at $800 \mathrm{~nm}$ was hermetically bonded to a clear plastic drape and sterilized (Medical Technique, Inc., Tucson, AZ).

\section{Preparation of Indocyanine Green Adsorbed to Human Serum Albumin}

ICG (25-mg vials) was purchased from Pulsion Medical Systems (Munich, Germany) and was resuspended in $10 \mathrm{cc}$ of sterile water for injection to yield a $2.5-\mathrm{mg} / \mathrm{ml}(3.2-\mathrm{mM})$ stock solution. Various amounts of this stock solution were transferred to a 50 -cc vial of Cealb (20\% human serum albumin [HSA] solution; Sanquin, Amsterdam, The Netherlands) to yield ICG in HSA (ICG:HSA) at a final concentration of $50 \mu \mathrm{M}, 100 \mu \mathrm{M}, 200 \mu \mathrm{M}, 400 \mu \mathrm{M}$, $500 \mu \mathrm{M}, 600 \mu \mathrm{M}, 800 \mu \mathrm{M}$, or $1000 \mu \mathrm{M}$. To obtain a final concentration of 800 and $1000 \mu \mathrm{M}$, ICG was resuspended in $5 \mathrm{cc}$ of sterile water, to yield a $6.4-\mathrm{mM}$ stock solution prior to dilution.

\section{Preclinical Characterization}

Animals were studied under the supervision of approved institutional protocol. Female Yorkshire pigs (E.M. Parsons and Sons, Hadley, MA) averaging $35 \mathrm{~kg}$ were induced with 4.4-mg/kg intramuscular Telazol (Fort Dodge Labs, Fort Dodge, IA), intubated, and maintained with $2 \%$ isoflurane (Baxter Healthcare, Deerfield, IL). Vital signs were monitored continuously. Excitation fluence rate for white light and 800-nm excitation light were 26,600 lux and 7.7 $\mathrm{mW} / \mathrm{cm}^{2}$, respectively. Also $100 \mu \mathrm{L}$ of the specified ICG:HSA solution was injected intradermally. ICG concentration of the ICG:HSA complex was systematically increased from 10 to $500 \mu \mathrm{M}$ to explore the counteracting effects of NIR fluorophore quenching at the injection site and NIR fluorophore dilution within the lymphatic channels.

\section{Dose-Optimization Clinical Trial}

Details of the initial 6-patient pilot study of breast cancer SLN mapping using ICG:HSA and Mini-FLARE is provided in the Supplementary Data. The 24-patient doseescalation clinical trial was approved by the Medical Ethics Committee of the Leiden University Medical Center and was performed in accordance with the ethical standards of the Helsinki Declaration of 1975. All patients who had planned to undergo a SLN procedure for invasive breast cancer or extensive high-risk carcinoma in situ were eligible for participation in the study. Patients had clinically negative axillary nodes as assessed by palpation and ultrasonography. Exclusion criteria were pregnancy, 
lactation, or an allergy to iodine, shellfish, or indocyanine green.

A total of 24 consecutive patients were included. All patients gave informed consent and were anonymized. Patients received the standard-of-care SLN procedure. For our institution, this implies 1 periareolar injection of approximately $\quad 100 \mathrm{MBq} \quad{ }^{99 m}$ Technetium-nanocolloid (mean \pm S.D. $=99.6 \pm 5.8 \mathrm{MBq}$ ) the day before surgery. Before the start of the operation, $1 \mathrm{ml}$ of patent blue $\mathrm{V}$ was injected peritumorally or periareolarly. Choice of the injection site was left to the surgeon. Directly after patent blue injection, 1.6-ml ICG:HSA was administered as 4 injections at the same location as the patent blue injections. After surgical scrub and sterile covering of the operation field, NIR fluorescence imaging was performed with the imaging head of the Mini-FLARE at approximately $30-\mathrm{cm}$ distance to the surgical field.

The surgical technique consisted of percutaneous assessment of NIR fluorescent signal in the breast and the axilla prior to skin incision. The location and length of the axillary skin incision was determined by the surgeon. Assessment of the surgical field using NIR fluorescence was applied continuously throughout the SLN procedure and surgical exploration. If the SLN was not easily detected by NIR fluorescence, the gamma probe was used to provide direction for surgical exploration. Camera exposure was between 5 and $250 \mathrm{~ms}$ as indicated. A SLN exhibiting a signal-to-background ratio (SBR) $\geq 1.1$ in situ was considered positive by NIR fluorescence. Background was chosen as an area within the surgical field, directly adjacent (within 1-2 cm) to the SLN.

Routine histopathological frozen analysis of SLNs was performed during surgery. If the patient participated in the After Mapping of the Axilla Radiotherapy or Surgery (AMAROS) trial and was randomized to the radiotherapy arm, no intraoperative frozen analysis was performed. SLNs were fixed in formalin and embedded in paraffin for routine hematoxylin, eosin, and immunohistopathological staining for AE1/AE3 at 3 levels, with an interval of 150-250 $\mu \mathrm{m}$, according to the Dutch guidelines for SLN analysis. Patients underwent an axillary lymph node dissection if the SLN was found to contain metastases, except for those patients who participated in the radiotherapy arm of the AMAROS trial. If isolated tumor cells (ITCs) were found $(<0.2 \mathrm{~mm})$, no axillary lymph node dissection was performed.

\section{Statistical Analysis}

For statistical analysis, SPSS statistical software package (Version 16.0, Chicago, IL) was used. Graphs were generated using GraphPad Prism Software (Version 5.01, La Jolla, CA). To compare the SBR between concentration groups, a one-way analysis of variance (ANOVA) was performed with pairwise comparison with least squares difference (LSD) adjustment for multiple testing. When the assumption of homogeneity of variance was violated (Levene's test), a $\log 10$ transformation was applied. All statistical tests were 2 -tailed and $P<.05$ was considered significant.

\section{RESULTS}

Design of the Mini-FLARE Imaging System and In Vitro Characterization

The Mini-FLARE imaging system (Fig. 1a) is composed of a small portable electronics cart and a counterweighted pole stand that supports the imaging head. Unlike FLARE, Mini-FLARE uses a flexible gooseneck arm, which permits positioning of the imaging head at extreme angles virtually anywhere over the surgical field. The only consumable for Mini-FLARE is a specially designed acrylic splash plate that is hermetically sealed to a plastic drape. Using sterile technique, the shield/drape is inserted into the imaging head, locked into place, and the drape is unfolded to encase the imaging head and imaging system pole stand (Fig. 1b). Optical light paths for white light (i.e., color video images) and the 2 semi-independent NIR fluorescence channels, 1 centered at $700 \mathrm{~nm}$ emission and the other at $800 \mathrm{~nm}$ emission, are shown in Fig. 1c.

Technical specifications of Mini-FLARE are detailed in Table 1. Of note, the working distance is up to $13 \mathrm{in}$. away from the patient, with field-of-view adjustable from 4.7 in. $(12 \mathrm{~cm})$ to 2 in. $(5 \mathrm{~cm})$ simply by moving the device toward or away from the surgical field. The cart occupies a volume of only $9.7 \mathrm{ft}^{3}$, and weighs only $272 \mathrm{lb}$. A 6-pedal footswitch and autofocus circuit permit hands-free operation. The cost of all parts is $\sim \$ 40,000$.

\section{Preclinical Optimization of NIR Fluorescent Lymphatic Tracers}

Our group has previously reported that ICG (and ICG:HSA) exhibits intense quenching (i.e., reduction of fluorescence emission) as its concentration is increased. ${ }^{15}$ That is, increasing concentration actually decreases fluorescence, so there would theoretically be no benefit to injecting high concentration for SLN mapping. This effect is demonstrated vividly in Fig. 2a (left), where the injection site becomes dramatically less fluorescent as concentration increases. As a general rule, if the concentration is high enough to see green color at the injection site, ICG fluorescence is severely quenched.

Importantly, we have recently discovered that dilution of the injected NIR fluorophore as it travels through the lymphatic system and mixes with lymph counteracts the 

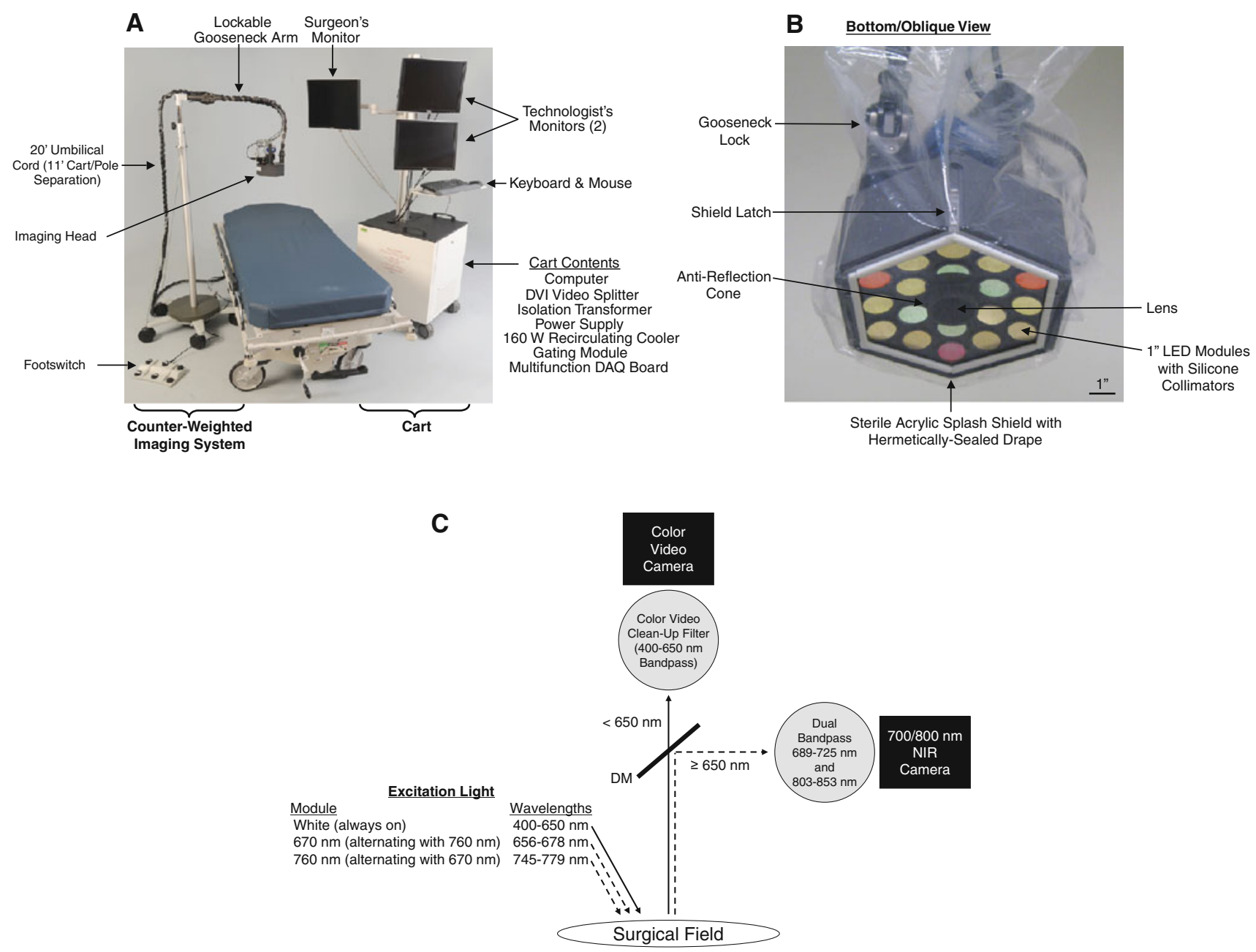

FIG. 1 The Mini-FLARE portable near-infrared fluorescence imaging system. a Imaging system, composed of electronics/monitor cart and counterweighted imaging system pole. b Sterile drape/shield attached to the imaging head with other major parts identified. c Excitation and emission light paths, and filtration for the MiniFLARE imaging system. DM, $650 \mathrm{~nm}$ dichroic mirror effect of quenching and results in signal in the SLN that increases with concentration, even when the injection site is barely fluorescent (Fig. 2a, right). This suggests that there will be an "optimal" concentration of injected NIR fluorophore such that final signal in the SLN is as high as possible.

\section{Optimization of ICG:HSA Dose in Women Undergoing SLN Mapping for Breast Cancer}

This study aimed to test the feasibility of NIR fluorescence in SLN detection, using ICG:HSA and the Mini-FLARE, in direct comparison with the conventional lymphatic tracers radiocolloid and patent blue. A total of 24 consecutive breast cancer patients underwent standardof-care SLN mapping with the addition of preoperative ICG:HSA injection and subsequent intraoperative NIR fluorescence imaging. Patient and tumor characteristics are provided in Table 2. Use of the Mini-FLARE during surgery did not interfere with the standard of care. Average time between ICG:HSA injection and skin incision was $16 \pm 3 \mathrm{~min}$ (Table 3 ). In all patients $(N=24)$, NIR fluorescence imaging enabled visualization of the SLN (Fig. 3). A total of 35 SLNs were identified, which were all radioactive and NIR fluorescent (Table 3 ). There were 5 SLNs from 4 patients that did not have blue staining from patent blue. In all patients, the NIR fluorescence signal in the SLN was detected before patent blue. Average time between skin incision and resection of the first SLN was $17 \pm 5$ min. After all nodes detected using NIR fluorescence were resected, the axilla was systematically inspected for any remaining radioactivity. No additional radioactive nodes were identified that were not detected by NIR fluorescence. No adverse reactions associated with the 
TABLE 1 Mini-FLARE imaging system specifications

\begin{tabular}{|c|c|}
\hline Category/specification & Description \\
\hline \multicolumn{2}{|l|}{ Physical } \\
\hline Size & Mobile cart: 24 in. $\mathrm{W} \times 24$ in. $\mathrm{D} \times 29$ in. $\mathrm{H}$; mast height: 76.5 in. \\
\hline \multirow[t]{3}{*}{ Weight } & Cart: $272 \mathrm{lb}$, including all electronics \\
\hline & Arm: $95 \mathrm{lb}$, including, stand, gooseneck, and imaging head \\
\hline & Imaging head: $8.8 \mathrm{lb}$ \\
\hline Arm and stand & $\begin{array}{l}\text { Flexible arm; reach: } 27-64 \text { in. from floor, } 42 \text { in. from center } \\
\text { of stand. Center of stand up to } 11 \text { linear } \mathrm{ft} \text { from cart }\end{array}$ \\
\hline \multicolumn{2}{|l|}{ Electrical } \\
\hline Voltage and plug & $120 \mathrm{~V}$ AC, $60 \mathrm{~Hz}$; single NEMA 5-15 $120 \mathrm{~V} / 15$ A AC plug \\
\hline Current & $5 \mathrm{~A} \max$ \\
\hline Grounding & Isolation transformer for all components; redundant chassis grounding \\
\hline Leakage current & $<300 \mu \mathrm{A}$ (per AAMI/IEC No. 60601) \\
\hline \multicolumn{2}{|l|}{ Sterility } \\
\hline Shield & Disposable acrylic shield with $\geq 95 \%$ transmission \\
\hline Drape & Disposable, custom-fit plastic drape bonded to shield \\
\hline \multicolumn{2}{|l|}{ Light source } \\
\hline Housing & Anodized aluminum with integrated liquid cooling \\
\hline Elements & Custom 25-mm circular LED arrays w/integrated linear drivers \\
\hline Electronics & Custom control board with embedded microcontroller \\
\hline Fluence rates & $\begin{array}{l}\text { 26,600 lux white light }(400-650 \mathrm{~nm}), 1.08 \mathrm{~mW} / \mathrm{cm}^{2} \text { of } 700 \mathrm{~nm}(656-678 \mathrm{~nm}) \text { excitation } 1 \\
\text { ight, } 7.70 \mathrm{~mW} / \mathrm{cm}^{2} \text { of } 800 \mathrm{~nm}(745-779 \mathrm{~nm}) \text { excitation light }\end{array}$ \\
\hline \multicolumn{2}{|l|}{ Optics } \\
\hline Working distance & $4-13$ in. $(10-32 \mathrm{~cm})$ from patient (reverse telephoto) \\
\hline Field-of-view & $12 \mathrm{~cm} \mathrm{~W} \times 9 \mathrm{~cm} \mathrm{H}$ at 13 in. working distance \\
\hline Emission/reflectance channels & $\begin{array}{l}\text { Color video (400-650 nm), } 700 \mathrm{~nm} \text { fluorescence }(689-725 \mathrm{~nm}), 800 \mathrm{~nm} \text { fluorescence }(800-848 \mathrm{~nm}) \text {, } \\
\text { all with simultaneous acquisition }\end{array}$ \\
\hline Pixel resolution & $640 \times 480$ for each camera \\
\hline System resolution & $320 \times 320 \mu \mathrm{m}(x, y)$ \\
\hline Display refresh & Up to $15 \mathrm{~Hz}$ simultaneous acquisition on both cameras \\
\hline NIR exposure time & Adjustable from $100 \mu$ s to $8 \mathrm{~s}$ \\
\hline \multicolumn{2}{|l|}{ Hands-free } \\
\hline Optics & Automatic focus \\
\hline Control & 6-pedal footswitch \\
\hline \multicolumn{2}{|l|}{ Monitors } \\
\hline Number & 3 cart-mounted 20 in.: 2 for operator and 1 for surgeon \\
\hline
\end{tabular}

use of ICG:HSA or the Mini-FLARE occurred. Two patients experienced a wound infection requiring antibiotics, and one patient underwent surgical re-exploration because of an expanding hematoma following axillary lymph node dissection (Table 3 ).

A second objective of this trial was to determine the optimal concentration of injected ICG:HSA for NIR fluorescence SLN mapping, i.e., which concentration provides the highest SBR in the SLN. Because ICG and ICG:HSA exhibit intense quenching as their concentrations are increased (typically above $50 \mu \mathrm{M}$ ), there would theoretically be no advantage to injecting higher doses of ICG:HSA. However, dilution of the lymphatic tracer occurs upon injection in the breast and uptake by the lymphatic system counteracting the quenching effect. To assess the relationship between the concentration of injected NIR fluorescent lymphatic tracer and final SBR in the SLN, patients were allocated to 8 ICG:HSA concentration groups ranging from 50 to $1000 \mu \mathrm{M}$. The concentration of ICG:HSA influenced the SBR and showed a normal distribution (Fig. 2b). The variances of the concentration groups were not equal (Levene's test, $P=.02$ ). A $\log 10$ transformation of the data could account for part of the unequal variances (Levene's test, $P=.05$ ). A 1-way ANOVA with pairwise comparison with LSD adjustment for multiple comparison showed that the SBRs of the 400, 
FIG. 2 Optimization of ICG:HSA dose as a function of the complex trade-off between fluorescence quenching at the injection site and dilution of fluorophore in lymphatic channels. a Preclinical studies in Yorkshire pigs. Subcutaneous injection sites (left; white arrows) showing quenching and resected SLNs (right) showing NIR fluorophore dilution for increasing concentrations of ICG:HSA. For each are displayed color video (left columns) and $800 \mathrm{~nm}$ NIR fluorescence (right columns) images obtained using $760 \mathrm{~nm}$ excitation light at $7.7 \mathrm{~mW} / \mathrm{cm}^{2}$. All camera exposure times were $45 \mathrm{~ms}$. Data are representative of $n=3$ pigs. b Optimization of ICG:HSA dose for breast cancer SLN mapping: Signal-tobackground ratio (mean \pm SD) of the SLNs (ordinate) as a function of injected dose of ICG:HSA (abscissa) in women undergoing SLN mapping for breast cancer. Statistical comparisons are: $200 \mathrm{vs}$. $400 \mu \mathrm{M}, P=.001 ; 200$ vs. $500 \mu \mathrm{M}, P=.001 ; 200$ vs. $600 \mu \mathrm{M}, P<.0001 ; 200$ vs. $800 \mu \mathrm{M}, P=.001 ; 1000$ vs. $400 \mu \mathrm{M}, P<.0001 ; 1000$ vs. $500 \mu \mathrm{M}, P<.0001 ; 1000$ vs. $600 \mu \mathrm{M}, P<.0001 ; 1000$ vs. $800 \mu \mathrm{M}, P<.0001$. The SBRs of the $400,500,600$, and $800 \mu \mathrm{M}$ concentration groups were not significantly different, although a trend was found favoring the $600 \mu \mathrm{M}$ concentration group (500 vs. 600, $P=.06$ )

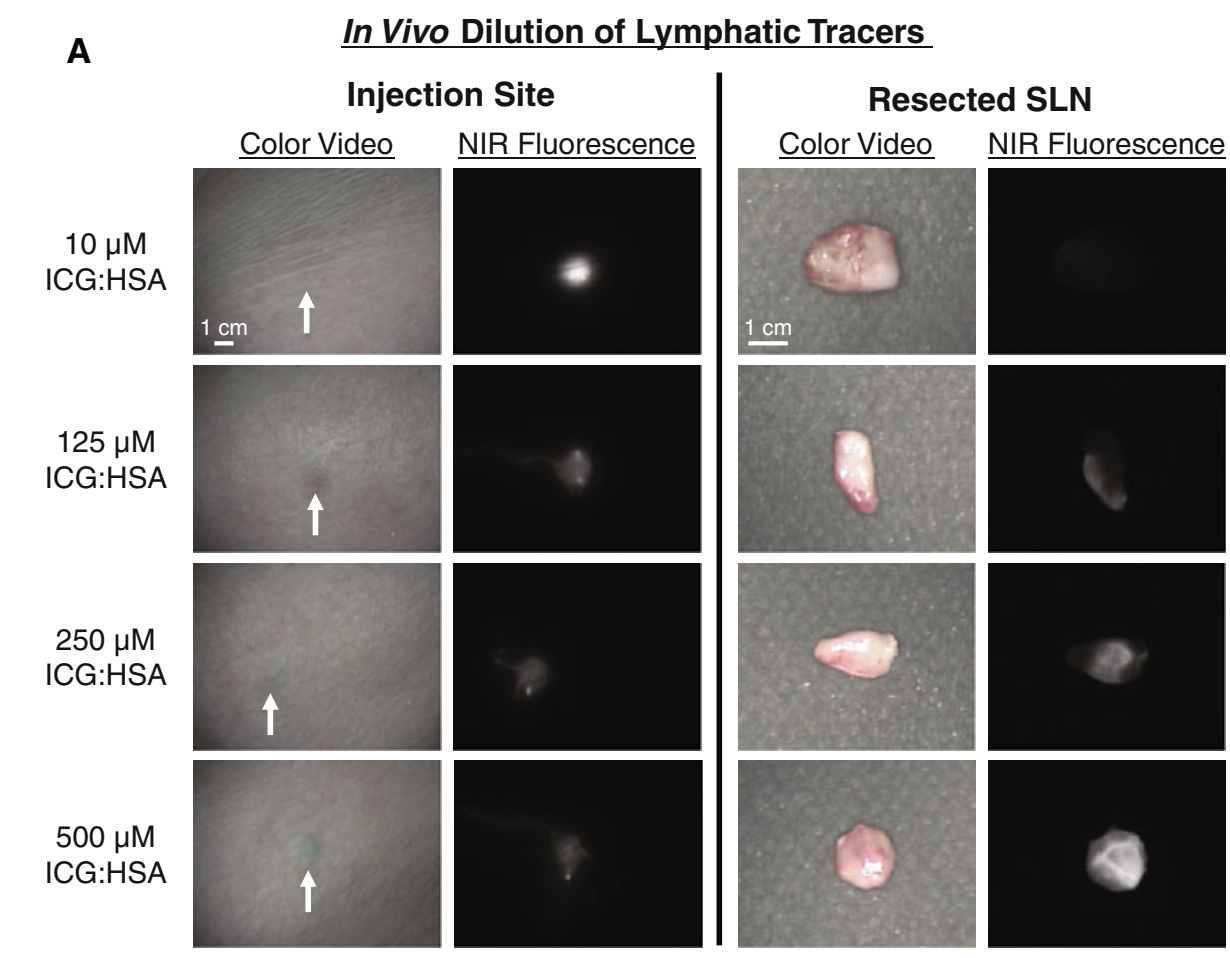

In Vivo Dilution of Lymphatic Tracers

B

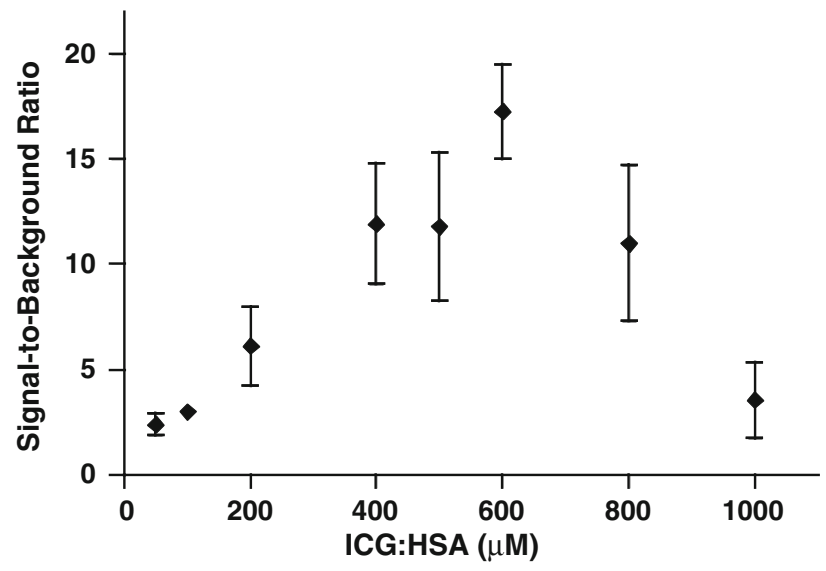

500,600 , and $800 \mu \mathrm{M}$ concentration groups were significantly higher than of the $50,100,200$, and $1000 \mu \mathrm{M}$ concentration groups (200 vs. $400, P=.001 ; 200$ vs. 500 , $P=.001 ; 200$ vs. $600, P<.0001 ; 200$ vs. $800, P=.001$; 1000 vs. $400, P<.0001 ; 1000$ vs. $500, P<.0001 ; 1000$ vs. $600, P<.0001 ; 1000$ vs. $800, P<.0001)$. The SBRs of the $400,500,600$, and $800 \mu \mathrm{M}$ concentration groups were not significantly different, although a trend was found favoring the $600 \mu \mathrm{M}$ concentration group (500 vs. 600 , $P=.06)$. The decline of the SBR in the $1000 \mu \mathrm{M}$ concentration group was caused by decreased NIR fluorescence signal of the SLN (800 vs. $1000, P=.001$ ), which suggests that quenching of ICG indeed occurred in the SLN.

\section{DISCUSSION}

Near-infrared (NIR) fluorescent light in the wavelength range of 700-900 $\mathrm{nm}$ is invisible to the human eye. It is also capable of penetrating millimeters into living tissue and is not obscured by autofluorescence. For these reasons, NIR fluorescent light is ideal for image-guided surgery. Indeed, several NIR fluorescence surgical imaging systems are already FDA approved or are in the process of obtaining approval (reviewed in Ref. ${ }^{17}$ ). The Mini-FLARE camera system used in this study is capable of displaying NIR fluorescence signal in relation to the surgical anatomy and illuminates the surgical field with white light. This enabled the surgeon to perform surgery under direct image guidance. 
TABLE 2 Patient and tumor characteristics

\begin{tabular}{|c|c|c|}
\hline Characteristic & $N$ & $\%$ \\
\hline Age (median, range) & $59.5(33-81)$ & \\
\hline \multicolumn{3}{|l|}{ Menopausal state } \\
\hline Premenopausal & 5 & 21 \\
\hline Postmenopausal & 19 & 79 \\
\hline Body mass index (median, range) & $25(18-38)$ & \\
\hline \multicolumn{3}{|l|}{ Skin type } \\
\hline II & 4 & 17 \\
\hline III & 20 & 83 \\
\hline Previous breast surgery $^{\mathrm{a}}$ & 3 & 13 \\
\hline Multifocality & 4 & 17 \\
\hline \multicolumn{3}{|l|}{ Tumor side } \\
\hline Left & 14 & 58 \\
\hline Right & 10 & 42 \\
\hline \multicolumn{3}{|l|}{ Tumor localization } \\
\hline Central & 5 & \\
\hline Lower inner & 1 & \\
\hline Lower outer & 1 & \\
\hline Upper inner & 6 & 25 \\
\hline Upper outer & 11 & 46 \\
\hline \multicolumn{3}{|l|}{ Type of operation } \\
\hline Ablation & 9 & 38 \\
\hline Wide local excision & 15 & 63 \\
\hline Pathological tumor size (median, range) & $15(3-35)$ & \\
\hline \multicolumn{3}{|l|}{ Histological type } \\
\hline Infiltrating ductal adenocarcinoma & 18 & 75 \\
\hline Infiltrating lobular adenocarcinoma & 2 & 8 \\
\hline Ductal carcinoma In Situ & 4 & 17 \\
\hline \multicolumn{3}{|l|}{ Histological grade } \\
\hline I & 3 & 13 \\
\hline II & 13 & 54 \\
\hline III & 8 & 33 \\
\hline \multicolumn{3}{|l|}{ Receptor status ${ }^{\mathrm{b}}$} \\
\hline $\mathrm{ER}+\mathrm{HER} 2-$ & 16 & 67 \\
\hline $\mathrm{ER}+\mathrm{HER} 2+$ & 3 & 13 \\
\hline ER - HER 2- & 1 & 4 \\
\hline Missing $^{\mathrm{c}}$ & 4 & 17 \\
\hline
\end{tabular}

a Previous breast surgery: silicone breast implantation, breast reduction, and re-excision

b HER2 status was determined using the chromogenic in situ hybridization (CISH) kit of Zymed (Invitrogen, Carlsbad, CA)

${ }^{c}$ Not applicable in 4 patients with ductal carcinoma in situ

NIR fluorescence optical imaging using Mini-FLARE offers the advantages of real-time, continuous, high-resolution, and high-sensitivity detection of SLNs, without the need for ionizing radiation. Because NIR light is invisible, there is no staining of the surgical field as with blue dyes, and the class of chemicals in which ICG belongs has a
TABLE 3 SLN identification results

\begin{tabular}{lll}
\hline Characteristic & $N$ & $\%$ \\
\hline
\end{tabular}

Injection site patent blue and ICG:HSA

$\begin{array}{lll}\text { Periareolar } & 20 & 83\end{array}$

Peritumoral $\quad 4 \quad 17$

SLN detection

Number of SLNs identified 35

Average number of SLNs identified (range) 1.45 (1-3)

Method of detection

$\begin{array}{lll}\text { Radioactive } & 35 & 100\end{array}$

Blue $\quad 30 \quad 86$

Near-infrared fluorescence $\quad 35 \quad 100$

Average time between injection of ICG:HSA $\quad 16 \pm 3$ and skin incision (SD) (minutes)

Average time between skin incision and SLN $\quad 17 \pm 5$ resection $(\mathrm{SD})$ (minutes)

Histology

$\begin{array}{lll}\text { Negative } & 26 & 74\end{array}$

Isolated tumor cells $\quad 3 \quad 9$

Micrometastases $\quad 0 \quad 0$

Macrometastases $\quad 6 \quad 17$

Axillary treatment

Axillary lymph node dissection $\quad 5 \quad 21$

Axillary radiotherapy $3 \quad 13$

Complications

\begin{tabular}{lll} 
No & 21 & 87 \\
Yes & $3^{\text {a }}$ & 13 \\
\hline
\end{tabular}

ICG:HSA indocyanine green adsorbed to human serum albumin, $S L N$ sentinel lymph node, $S D$ standard deviation

a Two patients experienced postoperative wound infection requiring treatment with antibiotics, and one patient underwent surgical re-exploration because of an expanding hematoma following axillary lymph node dissection

remarkable safety record in humans. ICG:HSA adds approximately $\$ 150$ to the cost of a case, but if future studies show that the blue dye and/or Tc-99 m sulfur colloid can be eliminated when using NIR fluorescence, a much larger savings will offset this cost. And, the surgeon can perform the injection procedure only minutes before SLN identification and resection. Although a technologist was used in this first in-human study, Mini-FLARE is equipped with hands-free operation and there is virtually no learning curve because unlike other SLN techniques, the lymphatic tracer can be visualized in real-time throughout the procedure.

An important objective of the study was to optimize NIR fluorescent contrast agent dose for breast cancer SLN mapping. The only clinically available NIR fluorescent lymphatic tracer, albeit approved for other indications, is ICG. ${ }^{7,11,13,18,19}$ We have previously proposed simple 
FIG. 3 Real-time NIR fluorescence imaging during sentinel lymph node mapping in women with breast cancer: Shown are typical in vivo (top row) and ex vivo (bottom row; postresection) results from a subject injected with $500 \mu \mathrm{M}$ ICG:HSA. White arrow identifies the SLN. NIR fluorescence (left) and pseudocolored (lime green) merge with the color video image (right). Exposure times were $50 \mathrm{~ms}$ for in vivo images and $30 \mathrm{~ms}$ for ex vivo images. $760 \mathrm{~nm}$ excitation fluence rate was $\sim 7.7 \mathrm{~mW} / \mathrm{cm}^{2}$ for all images. Scale bars indicate $1 \mathrm{~cm}$

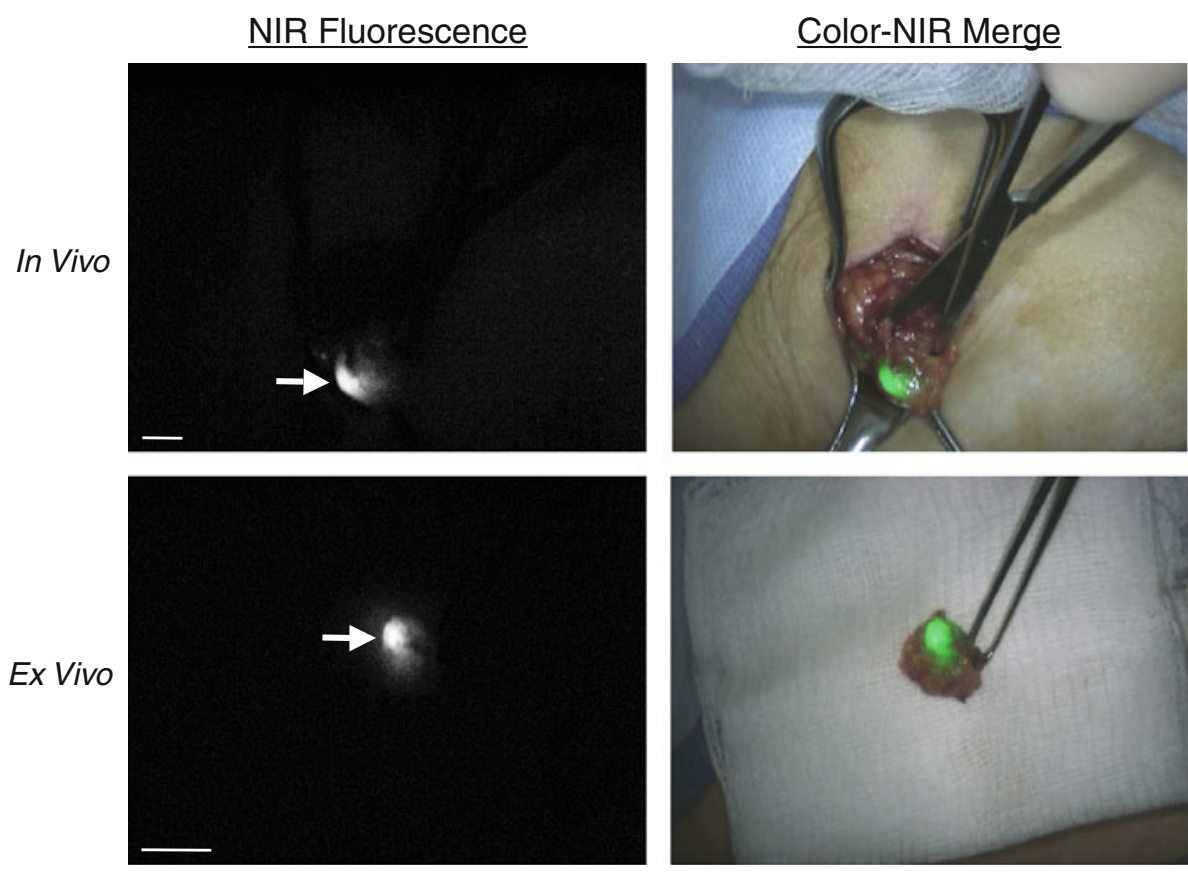

mixing of ICG and HSA (ICG:HAS) prior to injection because ICG is a small molecule and like blue dyes can pass through SLNs and exhibit a relatively low quantum yield in aqueous environments. ${ }^{14,15}$ Indeed, the product insert for ICG notes that it rapidly binds albumin when injected intravenously, so premixing merely improves kinetics. Importantly, though, premixing increases quantum yield (i.e., brightness) 3-fold and also results in a final hydrodynamic diameter $(\sim 7 \mathrm{~nm})$ that is better retained in the SLN. ${ }^{15}$

NIR fluorescent signal in the SLN is a complex function of the concentration of the injected NIR lymphatic tracer, the distance between the injection site and the SLN, the volume of ultrafiltrate within the lymphatic channels encountered by the NIR fluorophore, and retention of the NIR fluorophore by the SLN. Of these, only the injection concentration can be controlled. Our study suggested that an optimal ICG:HSA concentration is in the range 400 and $800 \mu \mathrm{M}$. In the $1000-\mu \mathrm{M}$ concentration group, the fluorescence intensity of the SLN and the SBR decreased rapidly, most likely due to quenching. Furthermore, since lymphatic vessels could be visualized percutaneously and directly after incision, particularly in the optimal dose range groups, a more efficient identification of SLNs was facilitated. This is of particular benefit for lymph nodes located deeper in the axilla, which exceed the 1-2 cm depth limit of the technology. These nodes could often be located by following the NIR fluorescent signal of the afferent lymphatic tract, as demonstrated in Supplementary Video 1.

The accuracy of SLN identification using ICG fluorescence was similar to that using conventional radioisotope scanning, as both techniques identified all SLNs and no additional fluorescent lymph nodes were identified. However, blue dye identification was not successful in 5 of 35 (14\%) SLNs. These results suggest that patent blue staining can be replaced by NIR fluorescence using ICG:HSA. Replacing patent blue has the additional advantage of absent tattooing of the breast and visual alteration of the surgical field. Moreover, the intrinsic dark color of patent blue dye can obscure the fluorescence intensity of any fluorescent lymphatic tracer. Indeed, in vitro tests using a fixed ICG:HSA concentration showed that addition of patent blue dye decreased NIR fluorescence of ICG:HSA (data not shown). An as yet unanswered question is whether radioisotope scanning can be omitted from the SLN procedure. A larger trial to address whether NIR fluorescence imaging alone can replace blue dyes and/or radiocolloids is ongoing.

In summary, this study demonstrated feasibility and accuracy of NIR fluorescence imaging using ICG:HSA and the Mini-FLARE imaging system for SLN mapping in breast cancer patients. The optimal dose of injected ICG:HSA lies between 400 and $800 \mu \mathrm{M}$ and can be chosen based on local preparation preferences. For example, in the United States, a dose of $500 \mu \mathrm{M}$ is most convenient since it requires minimal manipulation of albumin volumes.

ACKNOWLEDGMENT The BIDMC study team thanks Barbara L. Clough and Mireille Rosenberg for clinical trial preparation, Keith V. Belken from the BIDMC Investigational Pharmacy, Judith Hirshfield-Bartek for assistance with patient medical histories, Sunil Gupta for technical assistance with the imaging system, Lorissa A. Moffitt and Lindsey Gendall for editing, and Eugenia Trabucchi for administrative support. The Leiden study team thanks Gemma Ranke, 
Elly Krol-Warmerdam, Annemarie Voet-van den Brink, Gerlinda van Gent-de Bruijn (Breast Cancer Unit), and Linda van der Hulst (Central Pharmacy). Part of the study protocol was written during the 10th ECCO-AACR-ASCO Workshop on Methods in Clinical Cancer Research (Flims, Switzerland). We thank the following individuals and companies for their contributions to this project: Gordon Row (Yankee Modern Engineering), Kelly Stockwell and Paul Millman (Chroma Technology), David Comeau and Robert Waitt (Albright Technologies), Bob Zinter, Gary Avery, Phil Dillon, Will Barker, Craig Shaffer, and Ed Schultz (Qioptiq), Jeffrey Thumm (Duke River Engineering), Colin Johnson (LAE Technologies), Robert Eastlund (Graftek Imaging), John Fortini (Lauzon Manufacturing), Steve Huchro (Solid State Cooling), Clay Sakewitz, Johnny Fraga, and Will Richards (Design and Assembly Concepts), Ken Thomas and Fernando Irizarry (Sure Design), Paul Bistline and Phil Bonnette (Medical Technique, Inc.), Amy King (Civco), and Jim Cuthbertson (Nashua Circuits). This study was supported in part by the following grants from the National Institutes of Health (National Cancer Institute) to JVF: NIH Bioengineering Research Partnership grant No. R01-CA-115296 (JVF), Quick Trials for Imaging grant No. R21-CA130297 (JVF), Nuts Ohra Fund (ALV), the Maurits and Anna de Kock Foundation (ALV), and the American Women's Club of The Hague. JSDM is a MD-medical research trainee funded by The Netherlands Organisation for Health Research and Development (grant No. 92003526).

CONFLICT OF INTEREST STATEMENT Dr. J.V. Frangioni: All FLARE ${ }^{\mathrm{TM}}$ technology is owned by Beth Israel Deaconess Medical Center, a teaching hospital of Harvard Medical School. As inventor, Dr. Frangioni may someday receive royalties if products are commercialized. Dr. Frangioni is the founder and unpaid director of The FLARE Foundation, a non-profit organization focused on promoting the dissemination of medical imaging technology for research and clinical use. None of the other authors have any interest to disclose

Open Access This article is distributed under the terms of the Creative Commons Attribution Noncommercial License which permits any noncommercial use, distribution, and reproduction in any medium, provided the original author(s) and source are credited.

\section{REFERENCES}

1. Giuliano AE, Kirgan DM, Guenther JM, Morton DL. Lymphatic mapping and sentinel lymphadenectomy for breast cancer. Ann Surg. 1994;220:391-8.

2. Cox CE, Pendas S, Cox JM, Joseph E, Shons AR, Yeatman T, et al. Guidelines for sentinel node biopsy and lymphatic mapping of patients with breast cancer. Ann Surg. 1998;227:645-51.

3. Goyal A, Newcombe RG, Chhabra A, Mansel RE. Factors affecting failed localisation and false-negative rates of sentinel node biopsy in breast cancer-results of the ALMANAC validation phase. Breast Cancer Res Treat. 2006;99:203-8.

4. Krag DN, Anderson SJ, Julian TB, Brown AM, Harlow SP, Ashikaga $\mathrm{T}$, et al. Technical outcomes of sentinel-lymph-node resection and conventional axillary-lymph-node dissection in patients with clinically node-negative breast cancer: results from the NSABP B-32 randomised phase III trial. Lancet Oncol. 2007;8:881-8.

5. Straver ME, Meijnen P, van Tienhoven G, van de Velde CJ, Mansel RE, Bogaerts J, et al. Sentinel node identification rate and nodal involvement in the EORTC 10981-22023 AMAROS trial. Ann Surg Oncol. 2010;17:1854-61.

6. Zavagno G, De Salvo GL, Scalco G, Bozza F, Barutta L, Del Bianco P, et al. A Randomized clinical trial on sentinel lymph node biopsy versus axillary lymph node dissection in breast cancer: results of the Sentinella/GIVOM trial. Ann Surg. 2008;247:207-13.

7. Hirche C, Murawa D, Mohr Z, Kneif S, Hunerbein M. ICG fluorescence-guided sentinel node biopsy for axillary nodal staging in breast cancer. Breast Cancer Res Treat. 2010;121: 373-8.

8. Hojo T, Nagao T, Kikuyama M, Akashi S, Kinoshita T. Evaluation of sentinel node biopsy by combined fluorescent and dye method and lymph flow for breast cancer. Breast. 2010;19:210-3.

9. Kitai T, Inomoto T, Miwa M, Shikayama T. Fluorescence navigation with indocyanine green for detecting sentinel lymph nodes in breast cancer. Breast Cancer. 2005;12:211-5.

10. Murawa D, Hirche C, Dresel S, Hunerbein M. Sentinel lymph node biopsy in breast cancer guided by indocyanine green fluorescence. Br J Surg. 2009;96:1289-94.

11. Sevick-Muraca EM, Sharma R, Rasmussen JC, Marshall MV, Wendt JA, Pham HQ, et al. Imaging of lymph flow in breast cancer patients after microdose administration of a near-infrared fluorophore: feasibility study. Radiology. 2008;246:734-41.

12. Tagaya N, Yamazaki R, Nakagawa A, Abe A, Hamada K, Kubota $\mathrm{K}$, et al. Intraoperative identification of sentinel lymph nodes by near-infrared fluorescence imaging in patients with breast cancer. Am J Surg. 2008;195:850-3.

13. Tanaka R, Nakashima K, Fujimoto W. Sentinel lymph node detection in skin cancer using fluorescence navigation with indocyanine green. J Dermatol. 2009;36:468-70.

14. Troyan SL, Kianzad V, Gibbs-Strauss SL, Gioux S, Matsui A, Oketokoun $\mathrm{R}$, et al. The FLARE intraoperative near-infrared fluorescence imaging system: a first-in-human clinical trial in breast cancer sentinel lymph node mapping. Ann Surg Oncol. 2009;16:2943-52.

15. Ohnishi S, Lomnes SJ, Laurence RG, Gogbashian A, Mariani G, Frangioni JV. Organic alternatives to quantum dots for intraoperative near-infrared fluorescent sentinel lymph node mapping. Mol Imaging. 2005;4:172-81.

16. Gioux S, Kianzad V, Ciocan R, Gupta S, Oketokoun R, Frangioni JV. High-power, computer-controlled, light-emitting diode-based light sources for fluorescence imaging and image-guided surgery. Mol Imaging. 2009;8:156-65.

17. Gioux S, Choi HS, Frangioni JV. Image-guided surgery using invisible near-infrared light: fundamentals of clinical translation. Mol Imaging. 2010;9:237-55.

18. Kusano M, Tajima Y, Yamazaki K, Kato M, Watanabe M, Miwa M. Sentinel node mapping guided by indocyanine green fluorescence imaging: a new method for sentinel node navigation surgery in gastrointestinal cancer. Dig Surg. 2008;25:103-8.

19. Yamashita SI, Tokuishi K, Anami K, Miyawaki M, Moroga T, Kamei M, et al. Video-assisted thoracoscopic indocyanine green fluorescence imaging system shows sentinel lymph nodes in nonsmall-cell lung cancer. J Thorac Cardiovasc Surg. 2011;141: $141-4$. 